\title{
Introduction
}

\section{Echocardiography 2003}

W

are delighted to present the Heart Echo supplement for 2003 in conjunction with the British Society of Echocardiography. This year's supplement is based around a theme of "assessing left ventricular function". Once again we have tried to gather an "array" of international authors and I am sure you will agree that not only have we succeeded, but also that each article is of the very highest standard. The five articles cover a wide range of topics around this important area and I hope you find them instructive and entertaining.
Comprehensive and authoritative appraisal of some established techniques for assessment of systolic and diastolic function are combined with new technology as well as the relationship of echocardiography to biochemical assessment of left ventricular dysfunction using BNP. This has resulted in a broad overview of a hugely important subject to echocardiographers and clinicians alike. Although specifically targeted at the members of the British Society of Echocardiography, I am sure that anyone with an interest in assessing left ventricular function will find the articles of value.
On behalf of Heart, I would like to thank the British Society of Echocardiography for their assistance and cooperation, particularly Antoinette Kenny and Petros Nihoyannopoulos, without whom the supplement would not have been possible. In addition, we are extremely grateful to the sponsors listed below in allowing this supple ment to proceed to publication. Again, without them it would not have happened.

This supplement was made possible with sponsorship from Philips Medical Systems, Siemens Medical Solutions, Sonosite Limited, and Toshiba Medical Systems 